\title{
3 Ethical Aspects and Cultural Sensitivity in Language Revitalization
}

\section{Joanna Maryniak, Justyna Majerska-Sznajder, and Tymoteusz Król}

Ethics is often broadly understood as answering the questions of 'what is good?' and 'what is bad?'. Of course, this assumes that there are simple answers to these (and similar) queries. We define ethics in language revitalization as reflection upon the problems of correct, desirable, and sensitive actions around revitalization itself. Its importance stems from the fact that decisions taken without considering such ethical questions might end up being problematic or even harmful to communities. Thus tackling this issue can contribute to the success of language revitalization. While engaging in language revitalization, we ${ }^{1}$ often find ourselves in situations that are anything but obvious - for example, in situations where two (or more) possible good courses of action clash. For this reason, we will outline a series of discussion points for those engaged in revitalization. We have presented suggestions only where we felt we could offer potentially useful solutions or 'best practices'. Still we know that there is no valid and universal code of ethics or rules of conduct, even within a single community. For this reason, all of our points are, first and foremost, suggestions and points of departure for reflection.

\section{Basic Questions}

The first logical step to language revitalization is recognizing that a language is endangered. However this might not sit well with the community that speaks it (or that has historically spoken it). As the awareness that their language and culture is disappearing can be a depressing thought, some speakers may deny that it is happening. Therefore some people might ask whether to start a revitalization project at all? Other chapters in this book discuss reasons and benefits of language diversity and language revitalization (see Chapters $1,8,9$ ), so they might provide a starting point for such a discussion within a community.

1 This chapter is cowritten by two Indigenous Vilamovian activists and researchers and an external researcher collaborating with them. 
This however leads us to the second question: who should start (and continue) the revitalization process? Our experience shows two main kinds of people who are normally involved: internal (often called 'activists') and external, mostly researchers, who usually become interested in the language for purely academic reasons (like documenting interesting topics) and only later become involved in the revitalization process. These two groups differ in many ways. Their knowledge about the community is different and the time and money available to them, likewise, vary. Broadly speaking, activists are more likely to have more time and flexibility (since they often live in the community and so, even with full-time work, are still at hand) but less likely to have money at their disposal than researchers. On the other hand, researchers might have impressive financial means (thanks to funded projects) but strict time constraints (due to project deadlines). This can be a cause of misunderstandings and problems. Yet the process of revitalization doesn't end with those two groups: local authorities, politicians, and governments are often important as well. There might also be some overlap in that activists can become researchers (like two of the authors of this chapter, who are currently writing their $\mathrm{PhD}$ theses on various topics related to their home community of Wilamowice). Furthermore a researcher might become so engaged with the community that their actions cross over into activism. However no matter how respectable the authorities helping you, no matter how much money they can offer, no matter how good the methods they employ are, revitalization won't work without local engagement and local conviction about the goal, purposes, and benefits. Likewise, it will not succeed without respecting a local sense of ethics and good practices.

\section{A Decision-Making Process and Dealing with Dilemmas}

An Indigenous community itself is never homogenous and there might be people who disagree with the need for revitalization at all. An even bigger issue arises when only a part of the community wants to revitalize their language and the other part is against it. So, if someone wishes to start a revitalization project, would it make sense to try to convince them not to do so, or is it better to just ignore them and work on one's (self)-appointed tasks? As languages are usually lost because people decide not to speak them or cannot speak them anymore, an important challenge for activists is to question majority attitudes and practices within the community.

On the other hand, other community members might see revitalization as needed and useful but might not want to collaborate with others who are involved. Being an activist means being more visible than other members of the community. More often than not activists aren't democratically elected 
and this might put them in conflict with others - especially those who like being in the limelight or have been in a position of power for a long time. Opinions on the language itself can also vary as some activists are more concerned about taking the language forward and some might want to preserve older forms of it and keep it as it was previously. Of course, there may also be conflicts about how to go about revitalization.

\section{Coming from Outside and Dealing with Internal Parties}

What if a community at large doesn't want revitalization at all and the only interest is from outsiders, often (but not exclusively) researchers? Outsiders cannot revitalize a language without community involvement. All they can do is raise awareness and change attitudes, but this doesn't mean they can't try their best to promote the idea of revitalization in the community. Researchers face their own set of questions. How much and in what way should outsiders learn about the community beforehand? One thing we can emphasize is that coming to a community without any knowledge of local culture, traditions, and language will likely discourage its members from collaborating in the proposed project. Thus putting in time and effort during the preparation stage is essential when establishing collaborations. We will describe this in more detail in the sub-section entitled 'Establishing Collaboration'.

An unsolvable dilemma is whether a researcher should consult the community when planning a project before asking the funding agency for money. To make sure that the planned activities do not clash with the community's ideologies, needs, and behaviors, it is best to discuss the project with members of the community beforehand - even if with just a few. However, then their hopes might be raised and they might not understand the complicated procedures and long times needed for the projects to be reviewed and chosen for funding. This could lead them to think you let them down when you are only waiting for the results of various calls for proposals. One way not to run into this problem is not to discuss it at all, but then it might turn out that the planned actions don't sit well with the community. The best course of action may be to under-promise initially (i.e. promise the bare minimum needed so as not to raise the hopes and expectations either of the community or the funding agency) and then overdeliver once the project has started (don't stop at what you promised, give back as much as possible).

Time constraints posed by academic programs are something that should also be considered carefully. While the length of the project does not have to translate into the length of engagement with a community, it might determine it - especially if the community is far removed from the researcher's residence. In general, we have seen that Indigenous communities tend 
to warm up to outsiders gradually and at different paces. Usually they become more open and welcoming once they get to know them better. Therefore planning a longer project (or longer engagement) might help to address better the situation and goals of the community. What counts most in our opinion is that personal engagement outlasts the funding process.

Yet the best-case scenario is not always possible and there might be situations in which it is known from the start that the engagement will end after a certain period of time. In such a situation the best thing to do is to inform the members of the local community about that from the start and keep information clear and up to date during the communication process; sometimes saying something just once might prove not enough due to cultural differences. After receiving funding to work on one language and with a specific community, some researchers might be tempted to publish the results and move on to a new one. This is often referred to as a 'data extraction' approach and its ethical aspects can be problematic, especially considering community involvement and how it might benefit them. It is also important for outsiders to care about what will happen to their local collaborators once they leave. If a researcher left a good impression, all is well. But if not everything went right, members of the community who collaborated on the project might be associated with the researcher and thus reflected negatively on. It is a good idea to stay in constant contact with them and to do one's best to leave the best impression. In such a case, it is also advisable to plan to train local activists/volunteers to continue the revitalization activities once the researcher has left. These matters relate to the broader question of establishing collaboration, which we will discuss now.

\section{Establishing Collaboration}

How to establish initial collaboration with a community? How does one choose local collaborators? Is there a way to make sure that language revitalization goes on without discriminating any group in the community and making sure that all members have equal chances to be involved rather than marginalized? These questions guide decisions about establishing collaborations. First of all, it is advisable for external researchers coming to the community to include its members and make them part of the decision-making process at all stages of the project, including planning, realization, and evaluation. Local revitalizers are often in the best position when it comes to including and consulting other community members during all the planning and decision-making stages of the revitalization process. On the other hand, a naive external researcher may unwittingly ally with one local faction and alienate others. 
Creating a hierarchy as an outsider working with coworkers from the local community can cause controversies. This means, for example, avoiding favoritism, e.g. when commissioning handicrafts from specific people in the community. The eldest are not necessarily speaking the 'only correct' variant of the language or even a 'correct' one at all, as their language might have experienced attrition due to disuse. Those who do not know the language well might nevertheless have traditional knowledge, which is an important part of the culture. Local activists are not the only ones who can collaborate with researchers. Striving to understand relations among different groups of interest in the community, and remembering that sometimes the interested parties may try to hide these relations for various reasons, can prevent certain problems from arising. In some contexts, being an obvious outsider can help to avoid being associated with any faction.

Local revitalizers often act as 'radars', paying attention to the emotions, opinions, and attitudes of community members. But at the same time they might act against the 'common opinion' shared by many community members. During their activities they might tread 'uncomfortable' ground for other members of their community. This discomfort might be related to historical traumas or specific situations that the group or individuals have gone through. Local revitalizers might therefore be tempted to avoid certain subjects; caring for the well-being of community members can help the revitalization process gain more allies. However, confronting such topics with as much tact and caution as possible so as not to hurt anybody further might also prove a valuable experience. Local revitalizers are also in the most comfortable starting position when it comes to including the community during all the planning and decision-making stages of the revitalization process. However, they also should pay attention not to exclude any sections of the community - this means inviting everyone to get involved (and especially those who are interested). Worsening local conflicts or driving divisions deeper as a result of revitalization activities is precisely something that should be avoided. Local revitalizers have yet another advantage in that they have known the members of the community for a long time and so can choose their collaborators more thoughtfully and on the basis of longer experience.

In the case of many disappearing languages, the main language carriers are the elderly. When working in language revitalization, one must be careful not to exploit them - for example, through taking up too much of their time or reminding them too much of traumas they might have experienced. As they grow older, their health can worsen and they can eventually die, thus leaving younger people as possible collaborators. This can be emotionally difficult for researchers and activists who have got to know them. However, younger generations can't always help in the way 
that elders have done. If language revitalization is supported by a project with strict deadlines, taking care not to plan too much work for the elderly is even more salient, as their illness or death could result in unnecessary pressures on all the surviving parts of the community.

There may also be members of the community who do not speak the language in question and do not show any particular concern about it. Still some forms that appear in their use of the dominant language might be influenced by the minority one. Similarly, a seemingly 'worse' knowledge of the dominant language might not be a sign of an uneducated and 'worthless' interlocutor, but a new and hitherto unexplored field of research - one of language contact in that community.

It is also good to remember class and gender issues in the community, which are best analyzed on a case-by-case basis. Sometimes publishing a book or creating a movie in which the members of one class or people of one gender will be more prevalent can result in controversies. Of course, it can also be done on purpose to help to create visibility of minorities within minorities, but then it needs to be done consciously, not haphazardly without considering the implications for the group.

The position of an outsider can also be exploited by the community collaborators. A feeling of prestige or the possibility of receiving some benefits from collaborating with the academy can result in conflicts and internal discrimination. The opposite is also possible. Members of some communities do not want to get engaged in collaboration regarding the language because they fear being seen as deriving private profit from a common good, i.e. the language. There are no clear answers and solutions to such problems and they are best approached on a case-by-case basis and with the most careful use of intuition, respect, and sensitivity.

As an outsider, preparing for language revitalization in a community is less about choosing the correct kind of technical equipment or having the proper monetary means as it is about learning. Making an effort before meeting to learn as much as possible about some of the most important subjects such as local internal dependencies, cultural codes, and history of the field and community, can make a huge difference. We recommend that outsiders learn about the community's culture and history, including its rules of respect and conduct. Developing sensitivity toward the community's needs, its perspectives, and its knowledge systems might prove more different than assumed. Cultural differences are not only in play when dealing with far-away countries. There are some places that have such a vast amount of literature on them that it is not possible to read it through in a reasonable amount of time, so it is understandable that one has to limit oneself. However, there are also places that have next to nothing written about them, or that have their last description dating from a long 
time ago, thus making it horribly outdated. Whatever the case, effort is of paramount importance as it shows respect to the community through not coming totally unprepared. However, in no case should one treat the written word as having absolute precedence over what you are told by the members of the community - things might have changed since the writing of the books, they might reflect an external point of view or they might reflect perspectives of only one specific group within the community (and, as already remarked, most communities are not homogenous).

Even when working with communities near the researcher's home area and doing one's best to develop sensitivity and awareness of local norms and rules of respect, one can still be perceived negatively. Being conscious of that is a good step toward approaching possible issues. These might be the result of internalized negative stereotypes or judgments from both the sides: Researchers might be seen as people from huge cities, or as representatives of the dominant culture (both characteristics that can be perceived negatively). However, their actions might also prove offensive to the community without the researcher being aware. In such a case neither side is at fault and understanding that helps in approaching issues with an open mind.

\section{Facing the Past and Dealing with Political Tensions}

Many communities come with a burden of past or present oppression, persecution, experiences of ethnic discrimination, marginalization, and related traumas. In such cases, the group may ask itself difficult questions about how much visibility they want; in the context of their history, including possible traumas, minoritization, and related persecutions, it is possible that the group may not want to draw attention to itself. Would bringing up a specific subject result in the community's discomfort, fear, or insecurity? Would reminding the public of some difficult issues reignite old troubles? Sometimes it might seem that the problem does not exist or has been solved long ago, only for it to come back in the least expected moment. It might be reasonable to discuss these issues with community members - if and how they wish difficult topics to be brought up or dealt with - as sometimes time is needed for a community to realize what would be best for its members and the future of the language. In such a situation helping to create safe spaces for discussing it in the community would significantly facilitate such deliberations.

For example, in 2018 the Vilamovian youth, who were engaged in language revitalization, wrote and staged a theater play dealing with the violent ethnic persecutions suffered by the eldest generations during and after the World War II. This was not well received by local authorities and 
was very stressful for the youths themselves, in part because of Poland's descent into conservative authoritarianism, which sees minorities as a threat to the territorial integrity and ethnic cohesion of the state. However, the decision to stage this play was a bottom-up one, taken by the young members of the community. Instead of intimidating them, the experience became a source of pride and empowerment for them. Were it done as an external initiative (e.g. by a researcher or an institution), the negative reactions might have been seen as lacking of sensitivity toward the community and putting its young members in risky and disturbing circumstances. Thus, we believe that certain, especially difficult, decisions should be made only by members of the community. Even if they represent just one of its groups, this does not deprive them of the legitimacy to act.

Collaborating as an academic with a minority may position an outsider politically, whether they want it or not. Linguistic minorities often overlap with ethnic minorities and this raises questions about their nationality and possible separatism. Basque and Catalan in Spain are two famous cases, but similar examples abound (in Poland a very similar situation surrounds Silesia). Journalists will undoubtedly ask you for your opinions about those subjects and more. Academics may be asked to testify about such difficult topics in person, or to pen scientific opinions that will carry more weight than any research paper because on their basis some parliaments might vote yes or no to a law giving a community the right to learn in its language, or to use it in their signage etc. Writing this sort of text carries a huge responsibility so it is best to be careful not to hurt the most vulnerable party, i.e. the minoritized community. When asked whether what they are speaking is a language, the best answer is the one respecting the perspective of the community. The absolute worst course of action is to use a term that will be understood as diminishing the importance of the language. 'Dialect', 'patois', even 'ethnolect' all can be seen as dismissive and imposing such external categories on the speech of community language can be seen as unethical or as a form of violence.

\section{Ownership, Consent, and Other Legal Issues}

Legal issues also fall under the subject of ethics. Laws change so much and so often that any set of precise recommendations we might include here would quickly become outdated. Therefore we will name topics, which do not depend on a particular wording of the law but touch upon legal issues in general. While doing language revitalization, one needs not only to familiarize oneself with the laws of the land and obtain all the necessary permissions from local authorities, but also - especially in the case of academics - to comply with the legal requirements in one's own institution, 
with regard to national and international laws (if applicable), or the requirements of one's funding agency. If the revitalization is supported by sponsors, agreeing on these rules is also an ethical question.

There are also situations where what is legal may clash with what is ethical. This refers, for example, to intellectual property and authorship rights. These are often violated when local collaborators are treated as 'informants' and not as partners and coauthors of research and related language revitalization programmes, including scientific and educational products. It is ethical to consult people before publishing their data or transcribed utterances and, if possible, offer them the position of coauthors or named contributors. What would seem ethical and culturally sensitive may, however, clash with national or international legal regulations. For example, research participants usually do not legally own their words once recorded. The recordings legally belong to the organization that employs the person creating them. However, this does not have to be a guiding practice for sensitive researchers. Whatever the ownership rights might be in the country where one is working, including the collaborators and interlocutors as coauthors or of a publication, or at least naming them as contributors (if they agree for their identities to be revealed in a specific publication), is a commendable practice.

No matter what the laws might be in the country, there might be parts of culture that do not incur any legal protection but that the community does not want to be copied or published. For example, the Vilamovians (both generally as members of an ethnic minority who are inhabitants of the town of Wilamowice, but also more specifically as members of a tightly knit dance ensemble) have a very distinct song and dance repertoire and see any other folk group performing parts of it as a cause for outrage. Similarly the Maasai had their handicrafts copied - not only by huge Western corporations but also by other nearby tribes. So, sometimes it would be more sensitive for researchers not to engage in disseminating content which could be copied without the community's consent. Even local activists may be wary of engaging in external language revitalization because they do not want to be accused of 'betraying the group's secrets' or selling the rights to the language or culture. Of course, a full application of this principle would prevent anything from being published, so consultations are a reasonable course of action.

Another legal issue arises when the commercial benefits of revitalization go to outside parties. This is often intertwined with cultural appropriation as in the case of various big name fashion designers 'copying' or 'being closely inspired by' various Mexican traditional costumes, but it can also occur on a smaller scale as in the case of Wilamowice. Some enterprising Poles living in the region have started selling materials based 
on Vilamovian cultural heritage. Yet the economic profits do not reach the community who feels that they own the moral rights to those materials.

It is unacceptable to publish anything without prior consent. Of course, it does not have to be given in writing. There are groups that are historically illiterate and calling them out on it is a very bad idea. Yet there are also groups, which have been in a problematic situation vis-à-vis various bureaucratic forms, which they were forced or coerced to sign. In these cases, asking them to declare their consent in writing might recall these traumatic memories and so it is much better to include the expression of consent at the beginning of a recording. This might sometimes be problematic for academics if a funding agency or their institution asks for a written consent. Even so, it would be advisable for a researcher to prioritize the comfort and well-being of local collaborators over formal requirements, and, if necessary, negotiate oral consent with the institutions they depend on. After all, this may contribute to a greater decolonization and sensibilization of those institutions.

Moreover consent, according to the United Nations Declaration on the Rights of Indigenous Peoples, should always be understood as FPIC: 'Free, prior, and informed consent'. In other words, it is valid only when it is given freely (sometimes also termed 'enthusiastically' i.e. without pressure), when it is given prior to an action deriving from it (so, for example, requesting permission to record someone after the recording has already taken place is unethical), and when the person who is asked for consent understands what they are agreeing to. It follows that both written consent forms and oral questions relating to consent should employ language that is easy to understand. This suggestion can also be helpful for other forms of communication with the community. However, precautions are not always enough, and we can find ourselves in a situation when a person who was recorded or provided some other kind of information asks for it not to be disseminated. It is easy enough to deal with this before the fact, but what to do when material created already (book, movie, etc.) prompts this person to withdraw their consent? Of course, there are perfectly legal answers here, but they might not be satisfactory to the member of the community and what to do in such a situation is an example of a serious ethical challenge.

One might sometimes be confronted with a moral dilemma connected to gathered data. Let's say one finds some older, good-quality recordings and wants to share them with the community. However, the first collaborator to hear the recording informs you that the person featured in the recording was implicated in many problematic actions in the community and being reminded of them in a language-learning material could turn people away from the language even faster. There are various points of view and various relationships and reputations here at stake, and it is by no means easy to 
balance them. Such a recording could be anonymized or, if it stays recognizable even after such a process, one possibility is to use such a recording for very short excerpts, like the pronunciation of single words in a digital dictionary.

Sharing the documentation (as it is also part of the language revitalization process - see Chapter 13) is also an ethical question. Recorded materials might not belong to the community according to the letter of the law, but it does not change the fact that their content originates with them. Therefore the community may wish to receive it back. Recordings can easily be transferred to a hard drive and left in the community as a sort of incipient local archive. Vocabulary gathered can be typeset as a dictionary - even using a simple word processor, printing it in a print shop, and binding it at home. What counts above all are the content and the gestures. Communities may appreciate these ways of giving back much more than seeing a fancy hardcover book published after a few years by a big-name publisher. The same applies to older sources (such as archival documents or old recordings) as they might be useful in language revitalization. Community members often do not have access to those materials, either because they do not know where to find them or because the archives demand exorbitant prices for access to the copies. In these cases, giving someone, for example, a copy of an old record or a photo of their grandmother can do wonders for the collaboration. In all cases, however, when making such materials available, it is commendable to publish them in a language that the community will understand - not necessarily in the dominant one.

Ethics is also concerned with the problem of respect toward outsiders by community members and activists. Academics and other outsiders also have their rights, dreams, and sensitivities and they may be engaged and committed individuals with the best of motivations toward the community. They may also be vulnerable human beings. An ethical collaboration should be developed in both directions, especially when the future of an endangered language and community's well-being are at stake.

The last, but by no means least important part of ethics in revitalization, is ethics toward oneself. The revitalization of Wymysiöeryś was something that the two Indigenous authors have for years treated as a 'higher thing,' having priority over all else. We often put revitalization before our private lives, sometimes working on it $24 / 7$, even losing sleep. If this is your position, we don't want to argue that you should step down and treat it just like a hobby (though if you already do so, it is also a viable position) but remember the ethics of working with yourself: respect yourself and do not burn yourself out as it won't help the revitalization in any way. A healthy, well-rested, and positively minded person can do more for revitalization than one who is struggling to maintain a healthy work-life balance. 


\section{FURTHER READING}

Austin, P. K. (2010). Communities, ethics and rights in language documentation. Language Documentation and Description, 7, 34-54. London: SOAS, University of London. www.elpublishing.org/docs/1/07/ldd07_03.pdf.

Dwyer, A. M. (2006). Ethics and practicalities of cooperative fieldwork and analysis. In J. Gippert, N. Himmelmann, and U. Mosel, eds., Fundamentals of Language Documentation: A Handbook. Berlin: Mouton de Gruyter, pp. 31-66. (Spanish version: Ética y aspectos prácticos del trabajo de campo cooperativo. In J. Haviland and J. A. Flores Farfan, eds. (2007). Bases de la documentación lingüística. Mexico City: Instituto Nacional de Lenguas Indígenas, pp. 49-89.)

Grinevald, C. (2003). Speakers and documentation of endangered languages. In P. K. Austin, ed., Language Documentation and Description 1, pp. 52-72. www.elpublishing.org/itempage/7

\subsection{Being a Helper: A Few Ethical Considerations for Conducting Research with Indigenous Communities}

\section{Aleksandra Bergier}

In this capsule, I'd like to share a few ethical lessons I learned while collaborating with the Indigenous researchers and the Friendship Centres (urban Indigenous community organizations) in Ontario, Canada, on various Indigenous knowledge and language-related projects.

I'm particularly grateful for an opportunity to enhance my understanding of what it means to be a helper. I don't use this word in a sense of being a white do-gooder within a Western charity model. Rather, I draw on the culturally grounded meanings this term holds for the urban Indigenous communities I had the privilege of working with. Being a helper means contributing to the well-being of the community with one's unique skills and knowledge. It is about being part of a community circle where individuals with a wide range of identities and ways of being in the world feel that they belong while sharing their gifts and working for the benefit of all. Navigating the role of a helper within the research context is never limited to using skills typically associated with the work of a scholar - gathering information, making sense of data, and writing down the findings. It involves many other skills and responsibilities. One of them is the willingness to listen deeply and carefully before rushing to speak your truth. Another is sensitivity to the needs of the community members and the ability to respectfully follow cultural protocols. An act as simple as helping to clean a venue before a community gathering or ensuring a knowledge keeper's appropriate transportation to and from event can go a long way.

I had one of my most enlightening research experiences as I was sitting at the kitchen table at one of the Friendship Centres. I was simply listening to women who shared incredibly complex stories about their work while helping to chop vegetables, greeting guests and serving food at the community feast. This, of course, was part of 
a research plan previously approved by the community. I gained more insights that day than during dozens of formal interviews.

In my experience, research done in a good way is grounded in long-term, reciprocal relationships with the knowledge keepers. It generates useful knowledge in a way that's accountable and addresses the needs of specific communities. Often this means planning the research outcomes in a way that's immediately beneficial to people, for example, designing data collection activities around community initiatives and including expenses for these initiatives in a research budget. Examples include funding a language immersion camp, a land-based experiential workshop, a video, a toolkit, a Pow Wow, or a community feast.

Finally, I learned that an integral part of a researcher's work is an ongoing examination of their positionality. To me personally, it means exploring my own cultural foundations as a Polish researcher - connecting to my country's past, looking into the history of my family, and understanding how my identity and social position impact my work. 